\title{
Sport Specialization and Lower Extremity Injury Incidence: A Retrospective Pilot Study among Female Collegiate Soccer Players
}

\author{
Janae Anderson', Breanna Lewenthal', Suk-Kyu Kim², Jupil Ko ${ }^{1 *}$ \\ ${ }^{1}$ Northern Arizona University, Phoenix, United States \\ ${ }^{2}$ Dongguk University, Gyeongju, Republic of Korea
}

Received: June 19, 2018

Accepted: July 17, 2018

Published online: July 31, 2018

\section{Keywords:}

Athletic performance

Injury prevention

Overuse injury

Single sport
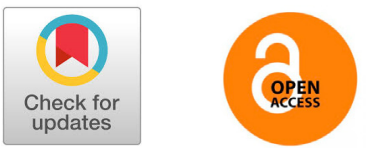

ORCID

Jupil Ko

http://orcid.org/0000-0003-1834-1113

\begin{abstract}
OBJECTIVES To assess the relationship between single sport specialization and lower extremity injury incidence among female collegiate soccer players.

METHODS Five participants ( 5 females; age $19.6 \pm 0.9$ years) were recruited from a Division I college soccer team. No other inclusion criteria were included. Injury incidence was recorded on a dichotomous scale. Presence of a lower extremity injury that resulted in greater than 2 days of missed practice indicated a 'yes' for injury incidence. Zero reported injuries and injuries resulting in less than 2 days of missed practice indicated a'no' for injury incidence. Sport specialization level was categorized on self-report questionnaire results. Participants were categorized as highly, moderately, lowly, or non-sport specialized.

RESULTS Each participant was highly sport specialized (100\%) and age of specialization ranged from 3 to 16 years old. Of the group total, three participants sustained a previous LEl that resulted in time-loss. An average of four days were lost due to injury.

CONCLUSIONS A sample of five, highly sport specialized, female soccer players lost an average of four days due to lower extremity injury.
\end{abstract}

(c) The Asian Society of Kinesiology and the Korean Academy of Kinesiology

\section{Introduction}

Intensive training in a single sport at the exclusion of other sports, termed sport specialization, remains a heavily debated topic [1-3]. Despite recent evidence that demonstrates an association between specialization and injury risk, rates of specialization and early specialization continue to rise [1-6]. The American Academy of Sports Medicine (AAOSM) [2] and

*Correspondence: Jupil Ko, 435 N. 5th Street, Northern Arizona University, Phoenix, AZ 85004, USA

E-mails:jupilko@gmail.com
American Medical Society for Sports Medicine (AMSSM) [3], among other organizations [3], discourage specialization and cite the potential of burnout [7], sports withdrawal [7], decreased motivation for participation [7], and increased rates of overuse injuries $[7,8]$ as cause to refrain. Prior to recent publications [9], conclusive data investigating the risks of specialization, specifically increased rates of overuse injury risk and/or incidence were limited $[2,4]$.

A study evaluating the dose-response relationship between sport activity and musculoskeletal pain revealed that for each hour per week of sports activity, there was a 3\% greater probability of development of musculoskeletal pain [10]. Although

(1) \$ This is an open-access article distributed under the terms of the Creative Commons Attribution License (http://creativecommons.org/licenses/by-nc/4.0/), which permits unrestricted use, distribution, and reproduction in any medium, provided the original work is properly cited. 
this study didn't specifically evaluate for the effects of sport specialization, it notes that excessive sport participation yields greater risk of pain development and potentially injury [10]. In another study, sport-specialized athletes experienced $12.1 \%$ more overuse injuries and $11.8 \%$ more serious injuries than non-specialized athletes [5]. McGuine et al. [9] found a direct association between specialization and lower extremity injury rates among co-ed high school athletes. Per stratification of specialization (high, moderate, low), the incidence of lower extremity injuries was highest for highly specialized athletes, followed by moderately specialized athletes [9].

Although prospective research was published, more retrospective sport-specific data is needed to begin determining which, if any, restrictions should be set for specialization degree and age. This study investigated the association between sport specialization and lower extremity injury incidence among collegiate Women's soccer athletes.

\section{Methods}

\section{Study Design and Setting}

Data were collected retrospectively at a Division I college during the 2017-2018 school year. A retrospective study (pilot) design was used to measure the incidence rate of lower extremity injuries (LEI) among non-, low, moderate, and high level sport specialized athletes. Each level of sport specialization, including non-specialized, were compared by LEI incidence rate. The sublevels of SS was low (LSS), moderate (MSS), and high level specialization (HSS). No inclusion or exclusion criteria were set for selecting the research setting.

\section{Participants}

A total of five female soccer players (5 female; age $19.6 \pm$ 0.89 years, height: $172.5 \pm 5.4 \mathrm{~cm}$, mass: $67.7 \pm 5.5 \mathrm{~kg}$ ) were recruited from a Division I college during a mid-season team meeting. Female soccer players medically cleared to play at the university during the 2017-2018 school year were eligible to be included. Potential participants needed to sign the informed consent document and HIPAA authorization form in order to participate. No exclusionary parameters were set for this study. All five of the recruits met inclusionary and exclu- sionary criteria. This study was approved by the Institutional Review Board (IRB ID\#1107457-1) at the given institution, and informed written consent was obtained from the participant prior to participation.

\section{Procedures}

Researchers scheduled a date and time to attend a mid-season team meeting to recruit participants. Recruits were informed of purpose of the research, time requirements for participation and potential risks and provided the emails of the researchers. Those interested were instructed to email the researchers for more information. The email included a brief overview of the study purpose, time requirements for the questionnaire, the Human Subject Informed Consent, and available dates and times to meet with researchers on campus, in the student-athlete center, to complete the questionnaire. The informed consent was provided for the athletes to read, they did not need to complete any portions prior to meeting with researchers. During the questionnaire completion meeting, researchers obtained signatures on two copies of the Human Subject Informed Consent and the HIPAA Authorization form. Signed informed consent, authorization forms, and questionnaires were scanned and saved onto a dual-password protected laptop and the original documents were shredded. Researchers read the questionnaire aloud and recorded participant responses on paper. The participant was able to review their answers prior to the conclusion of the meeting. Participants were assigned a code which was written atop the questionnaire. The coding system was memorized by the researchers, and therefore not written down in any location.

Medical history and physical examination information pertinent to lower extremity injury were obtained via pre-participation examination records on the NextGen Electronic Medical Record software utilized by the university sports medicine department. Researchers completed HIPAA training modules through the university health services department to obtain username and password access to the software. Data including age, weight, height, sport, previous lower extremity injury records, and time loss were ascertained from NextGen (Quality Systems Inc. Irvine, CA, Unit- 
ed States) and documented in an encrypted Excel spreadsheet. All information was coded, encrypted, and stored on a dual-password protected laptop.

\section{Questionnaire}

With the assistance of the research administrators, participants completed a paper questionnaire consisting of questions regarding sport participation, specialization classification and LEI history. Questionnaires were completed on campus and took approximately 10 minutes to complete. Researchers read the questionnaire aloud and recorded participant responses on paper. Each participant was asked to recall their age upon beginning sport, previous and current sport participation, months spent training and competing in those sports, and whether they quit other sports to focus on one. Athletes were categorized by degree of sport-specialization based on a specialization score they received from the following three self-reported questions: (1) 'Was one main sport more important to you than any other(s)?'; (2) 'Did you quit other organized and competitive sport(s) to focus on one main sport?'; and (3) 'How many months per year did you train for that sport?' A 'yes' response received 1 point, and 'no' responses received 0 points. Participants that reported $>8$ months of training for one sport in a year received 1 point, and those that reported less than 8 months training received 0 points. The points were summed to represent a participant's degree of sport-specialization, with $</=1,2$ and 3 representing low, moderate, and high degree of specialization, respectively. These three questions were chosen to stratify athletes by specialization degree; however, this questionnaire has not yet been validated. Although the questionnaire used in this study has not been validated, components regarding sport specialization identification have been utilized in various studies and settings [6-9]. Researchers also individually reviewed each question with the participant and injury reports were confirmed via electronic health records to make sure information was accurately recalled and recorded. For this study, LEI was defined as a musculoskeletal injury that occurred during sport participation and caused the athlete to seek treatment from a medical provider. LEI's were categorized according to the following extremity regions: upper leg, knee, lower leg, ankle, and foot. Information regarding date of occurrence, days missed, injury description and mechanism of injury were ascertained for each LEI. The mechanism of injury was classified as acute contact, acute noncontact, or overuse injuries.

\section{Statistical Analysis}

Raw data were recorded and analyzed in Microsoft Excel (Microsoft Excel, Version 15.0). Statistics including means and standard deviations, proportions (\%), was calculated. Mean and standard deviation were calculated for participants' age, age starting primary sport, and days lost due to injury.

\section{Results}

A total of five participants (5 female; age $19.6 \pm 0.89$ years) completed the questionnaire. Participant demographics are listed in Table 1. Each participant was highly sport specialized (100\%) and age of specialization ranged from 3 to 16 years old. Of the group total, three participants sustained a previous LEI that resulted in time-loss. An average of four days were lost due to injury among the group. The distribution of sport specialization level, age, and criteria of stratification are included in Table 2.

\section{Discussion}

Dialog concerning sport specialization is of great importance for athletes, adolescents in particular. Whether sport initiation is on behalf of the child, parent, coach, or other party, the reason for initiation should be discussed. Research shows most are under the impression that engaging adolescents in a single sport early on will increase the opportunity for upward progression, recognition, scholarship, and elite/

Table 1. Participants' Demographics Mean and Standard Deviation

\begin{tabular}{cccc}
\hline Age $(\mathrm{yr})$ & Height $(\mathrm{cm})$ & Mass $(\mathrm{kg})$ & BMI \\
\hline $19.65( \pm 0.89)$ & $165.97( \pm 7.79)$ & $61.48( \pm 4.71)$ & $22.31( \pm 2.64)$ \\
\hline
\end{tabular}


Table 2. Sport Specialization Stratification

\begin{tabular}{|c|c|c|c|c|c|c|}
\hline Participants & $\begin{array}{l}\text { Sport initiation } \\
\text { age (years) }\end{array}$ & $\begin{array}{l}\text { Did you quit } \\
\text { another sport } \\
\text { to focus on your } \\
\text { primary sport? } \\
(\mathrm{Y} / \mathrm{N})\end{array}$ & $\begin{array}{l}\text { Quitting age } \\
\text { (years) }\end{array}$ & $\begin{array}{l}\text { Did you consider } \\
\text { your primary sport } \\
\text { more significant than } \\
\text { others? }(\mathrm{Y} / \mathrm{N})\end{array}$ & $\begin{array}{l}\text { Did you train and/ } \\
\text { or compete in } \\
\text { your primary sport } \\
\text { for greater than } 8 \\
\text { months in a year? } \\
(\mathrm{Y} / \mathrm{N})\end{array}$ & $\begin{array}{c}\text { Overall sport } \\
\text { specialization level (HSS, } \\
\text { MSS, LSS, NSS) }\end{array}$ \\
\hline 1 & 5 & Y & 13 & Y & $\mathrm{Y}$ & HSS \\
\hline 2 & 3 & Y & 13 & Y & Y & HSS \\
\hline 3 & 12 & Y & 16 & Y & $Y$ & HSS \\
\hline 3 & 10 & Y & 18 & Y & $Y$ & HSS \\
\hline 3 & 4 & * & * & * & Y & HSS \\
\hline \multicolumn{7}{|c|}{${ }^{*}=$ Participant 5 began participation in a single sport at a young age and was not required to quit } \\
\hline \multicolumn{7}{|c|}{ HSS = High Sport Specialization } \\
\hline \multicolumn{7}{|c|}{ MSS = Moderate Sport Specialization } \\
\hline \multicolumn{7}{|c|}{ LSS = Low Sport Specialization } \\
\hline NSS $=$ Non-Sport Sp & tion & & & & & \\
\hline
\end{tabular}

professional status $[10,11]$. However, evidence exists to refute that proposal [11]. Additionally, research regarding longterm psychosocial and physical effects suggest that the practice may, in fact, be detrimental rather than beneficial $[7,11]$. Young athletes are required to dedicate significant amounts of time to year-round practicing, limiting their social interactions and potentially hampering scholastic achievements [4].

As the body matures, year-round, intense participation in a single sport can have negative physical effects; up to $50 \%$ of all pediatric sports injuries are due to overuse $[7,8]$. Our research indicated an average of four days were lost due to common overuse conditions. Conditions such as patellofemoral pain, Osgood-Schlatter Disease, and Sinding-Larsen-Johansson Syndrome are three common overuse conditions experienced by sport specialized athletes [6]. Researchers note a four times greater occurrence of these conditions in specialized athletes compared to multi-sport athletes [6]. Varying sport participation allows younger athletes to gain a variety of motor skills that can be applied across many sports and promote neuromuscular adaptation [12]. We recommend sport sampling in early athletic years to potentially counteract the occurrence of overuse injuries associated with early sport specialization. Well-rounded practice and conditioning programs can be used to incorporate various skills and movements for athletes.

Our results do not indicate a relationship between injury incidence and specialization, however, previous researchers with greater sample sizes found strong correlations [9]. There are limitations to the current study. The final sample size was only five total participants. The study was limited to female collegiate soccer players and therefore inference to other sport teams is limited. The stratification of sport specialization was based on self-reported answers to a standardized questionnaire, which may include misremembered information, omitted details, and response bias. Also, the specific type of injury was not collected in the current study due to the institution's regulation.

\section{Conclusions}

Intense, year-round participation in sport poses risks of injury. Sport specialization is a continued practice, and one that is initiated earlier in athletic careers than ever. Research 
analyzing potential long-term effects of sport specialization are needed to inform athletes, parents, and coaches as well as promote preventative measures for risks introduced through specialization.

\section{Conflicts of Interest}

The authors declare no conflict of interest. All authors indicate that no benefits in any form have been received or will be received from a commercial party related directly or indirectly to the participant of this article.

\section{References}

1. Intensive training and sports specialization in young athletes. American Academy of Pediatrics. Committee on Sports Medicine and Fitness. Pediatrics. 2000; 106:154-157.

2. LaPrade RF, Agel J, Baker J, et al. AOSSM early sport specialization consensus statement. Orthop J Sports Med. 2016; 4(4):2325967116644241.

3. Selected issues for the adolescent athlete and the team physician: a consensus statement. Med Sci Sports Exerc. 2008; 40(11):1997-2012.

4. McLeod TCV, Decoster LC, Loud KJ, et al. National Athletic Trainers' Association position statement: prevention of pediatric overuse injuries. J Athl Train. 2011; 46(2):206-220.

5. Pasulka J, Jayanthi N, McCann A, et al. Specialization patterns across various youth sports and relationship to injury risk. Phys Sportsmed. 2017; 45(3):344-352.

6. Hall R, Foss KB, Hewett TE, et al. Sport specialization's association with an increased risk of developing anterior knee pain in adolescent female athletes. J Sport Rehab. 2015; 24(1):31-35.

7. Malina RM. Early sport specialization: roots, effectiveness, risks. Curr Sports Med Rep. 2010; 9(6):364371.

8. Smucny M, Parikh SN, Pandya NK. Consequences of single sport specialization in the pediatric and adolescent athlete. Orthop Clin North Am. 2015; 46(2):249259.

9. McGuine TA, Post EG, Hetzel SJ, et al. A prospective study on the effect of sport specialization on lower extremity injury rates in high school athletes. Am J Sports Med. 2017; 45(12):2706-2712.

10. Post EG, Trigsted SM, Riekena JW, et al. The association of sport specialization and training volume with injury history in youth athletes. Am J Sports Med. 2017; 45(6):1405-1412.

11. Ericsson KA, Krampe RT, Teschromer C. The role of deliberate practice in the acquisition of expert performance. Psych Review. 1993; 100(3):363-406.

12. Myer GD, Faigenbaum AD, Ford KR, et al. When to initiate integrative neuromuscular training to reduce sports-related injuries and enhance health in youth? Curr Sports Med Rep. 2011; 10(3):157-166. 